\title{
Surface Modification of PEEK-WC Membranes by Wet Phase Inversion for Ni(II) Adsorption
}

\author{
Said Bey ${ }^{1}$, Mohamed Benamor ${ }^{1}$, Enrico Drioli ${ }^{2}$ \\ ${ }^{1}$ Membrane Process and Separation, Recuperation Techniques Laboratory, Faculty of Technology, Bejaia University, Bejaia, Algeria \\ ${ }^{2}$ Institute on Membrane Technology (ITM-CNR), Università della Calabria, Rende, Italy \\ Email: Saidbey06@yahoo.com
}

Received May 2, 2013; revised June 3, 2013; accepted June 19, 2013

Copyright (C) 2013 Said Bey et al. This is an open access article distributed under the Creative Commons Attribution License, which permits unrestricted use, distribution, and reproduction in any medium, provided the original work is properly cited.

\begin{abstract}
This work focuses on the preparation and characterization of flat sheet membrane based on modified polyether ether keetone (PEEK-WC). Additives, such as dibutyl phatalate (DBP) and diethyl hexyl phosphoric acid (DEHPA), were used to investigate their effect on membranes properties which are prepared by immersion precipitation. For that, several techniques were used to characterize membranes like thermal analyses, scanning electron microscopy and microanalyses. SEM pictures show versatile structures of the membranes from dense to porous membranes characterized by a sponge and finger like structure. Moreover, microanalyses of both surfaces, bottom and top surfaces show an aggregation of DEHPA at the top surface of the membrane. However, by adding dibutylphtalate, a well dispersion of the extractant was observed. Initially, micro-porous membranes were used in supported liquid membranes experiments for $\mathrm{Ni}(\mathrm{II})$ metal ions transports using diethyl hexyl phosphoric acid (DEHPA) as carrier. The extraction efficiency was very low about $28 \%$, but enhanced by adding xylene to the organic phase. However, the modified membranes (with additives) by DBP and DEHPA were used on solid liquid extraction of Ni(II). The results show that by adding the plasticizer and the extractant, the efficiency of the system reached $63 \%$.
\end{abstract}

Keywords: DEHPA; Phase Inversion; PEEK-WC; Ni(II); SLM

\section{Introduction}

Up to now, the majority of synthetic polymeric membranes are produced by the phase inversion process where a thin layer of polymer, dissolved in an appropriate solvent, is casted on suitable support and phase separation is introduced by a non solvent [1-3]. The final membrane structure depends on several experimental parameters: the composition of polymer solution (additives, solvent, concentration), temperature, etc. In general, additives, with low molecular weight, are used in order to affect the morphology of the membrane or as pore former such as polyvinyli pyrrolidone (PVP), polyethylene glycol (PEG), maleic acid [4-7]. However, a large number of additives with polymers that can possibly be used remain unexplored. Hence, the aim of the present work is to investigate the effect of the dibutyl phthalate (DBP), used as plasticizer, and diethyl hexyl phosphoric acid (DEHPA) on the properties and surface modification of membranes based on a modified polyether ether ketone (PEEK-WC) prepared by wet phase inversion. A microanalysis of both surfaces, the top and the bottom surfaces, is introduced to understand the displacement of the components. The virgin micro-porous membranes were used for the transport of Ni(II) by supported liquid membrane and DEHPA as carrier and a modified one, DEHPA and DBP were used for solid-liquid extraction of $\mathrm{Ni}(\mathrm{II})$.

\section{Experimental}

\subsection{Membrane Preparation}

For membrane sample preparation, 14\% (wt) of the PEEK-WC polymer $\left(M \mathrm{w}=224 \mathrm{~kg} / \mathrm{mol} ; \mathrm{Tg}=225^{\circ} \mathrm{C}\right.$; Chang Chung institute-China) has been dissolved in dimethyl acetamide (DMAc, Fluka). The carrier contained in the membrane is an organo-phosphoric acid derivative with plasticizing properties. After complete dissolution of the polymer, the DBP (DBP; bp: $340^{\circ} \mathrm{C}$, PROLABO) or DEHPA (DEHPA, bp: $200^{\circ} \mathrm{C}$, SIGMA) (or mixture of them) was added to the polymer solution. DBP is used, as plasticizer, due to its compatibility with several polymers and for a high dispersion of the extractant (DEHPA) in polymer matrix. Then the mixture was 
vigorously stirred to merge the components homogeneously and the mixture was casted by a casting knife (casting thickness $250 \mu \mathrm{m}$ ) on glass plat to induce coagulation by immersion precipitation in a water coagulation bath. The experimental conditions of membrane preparation are reported in Table 1. The prepared membranes were dried in an oven overnight at $60^{\circ} \mathrm{C}$ for further analysis.

\subsection{Scanning Electronic Microscopy}

The Scanning Electron Microscopy (FEI QUANTA 200) at $20 \mathrm{Kv}$ was used to study the morphology of the membranes. For cross section analysis the membrane samples were freeze fractured in liquid nitrogen. The chemical composition of the top and bottom surfaces, of the modified membranes, were analyzed by energy dispersion X-ray analysis (EDS) instrument (EDAX).

\subsection{Thermal Analysis TGA}

Thermo-gravimetric analysis (TGA) was carried out for the polymer and for the modified membranes (polymer with additives DBP and DEHPA) with SETARAM TG6DTA92 in nitrogen.

\subsection{Extraction of Ni(II) by Supported Liquid Membrane and Solid-Liquid Systems}

The supported liquid membrane is carried out by the impregnation of an asymmetric micro-porous PEEK-WC membrane by $40 \%$ (volume) DEHPA diluted in a mixture of xylene and heptane. The extracting module consists of a two-compartment cell with an effective membrane area of $9 \mathrm{~cm}^{2}$. The procedure of the extraction is the same used in our previous work [8]. The flow of the two solutions was about $5.54 \mathrm{ml} / \mathrm{min}$ (MASTERFLEX pump $7418)$ and equal volume of samples $(0.2 \mathrm{ml})$ was withdrawn from both compartments at desired time interval. The Ni(II) concentrations were determined by using analytical kits (Carlo Erba reagent), based on a colori-

Table 1. Overview of membrane preparation conditions: coagulation bath: distilled water; initial polymer concentration: $14 \%$ (wt) in dimethyl acetamide (DMAc); temperature $25^{\circ} \mathrm{C}$.

\begin{tabular}{cccc}
\hline $\mathrm{N}^{\circ}$ & Polymer (wt\%) & DBP (wt\%) & DEHPA (wt\%) \\
\hline A & 100 & 0 & 0 \\
B & 60 & 0 & 40 \\
C & 60 & 20 & 20 \\
D & 50 & 20 & 30 \\
E & 40 & 20 & 40 \\
F & 80 & 20 & 0 \\
\hline
\end{tabular}

metric reaction and absorbance reading at $442 \mathrm{~nm}$ wavelength. A UV-1601 (SCHIMADZU) recording spectrophotometer was used for absorbance readings.

Solid-liquid extraction of $\mathrm{Ni}(\mathrm{II})$ by the modified membranes is carried out in a batch system with $100 \mathrm{mg}$ of small pieces of modified membranes in contact with 100 $\mathrm{ml}$ of aqueous solution containing $10 \mathrm{ppm}$ of $\mathrm{Ni}(\mathrm{II})$. The system is kept under stirring (600 rpm) for 24 hours.

\section{Results and Discussion}

\subsection{Morphology Analysis}

The morphology of the membrane plays a fundamental role in the transport of ions through the matrix. The SEM pictures of PEEK-WC/DBP/DEHPA membranes are shown in Figure 1.

From the SEM pictures we note that the presence of additives affect considerably the morphology of the membranes. Excepting for the membrane (B) where the two surfaces are smooth, very high asymmetric membranes were obtained characterized by a smooth top surface, with some aggregates due to the additives, and highly porous bottom surface. However, the analysis of the cross section of the different membranes shows the formation of macro-voids with different shape and form due to a low concentration of the polymer.

In the case of the presence of DEHPA (membrane B) alone, the membrane present internal cavities delimited by two dense layers due essentially to liquid-liquid demixing process. The cavities are smaller and not longed the cross section compared to the cavities in the case of the membrane A. The presence of DEHPA reduces the rate of demixing and eventually the macro-voids formation. Contrary, the presence of DBP (membrane F) produces an asymmetric dense membrane with large macrovoids.

The presence of the two additives together, the macrovoids became less numerous, large and tend to disappear (membrane C). However, the high concentration of DEHPA (40\% wt) induced the formation of a sponge-like structure with small pores in the cross section supported by macro-porous sub-layer (membrane E).

Microanalysis of the phosphorus in the bottom and the top surface, contained in diethyl hexyl phosphoric acid, shows a very high concentration of this element in the top surface (Table 2) for the membranes B, C and D. The absence of the plasticizer DBP (membrane B) enhanced the aggregation of the DEHPA in the top surface (11.5\%). However, its presence allows the dispersion of the DEHPA in the two surfaces. This can be explained by the interaction between the DEHPA and DBP. It seems that, in the absence of DBP, DEHPA aggregate in the top surface of the membranes influenced by the exchange between the solvent and the non-solvent, by reducing it's 

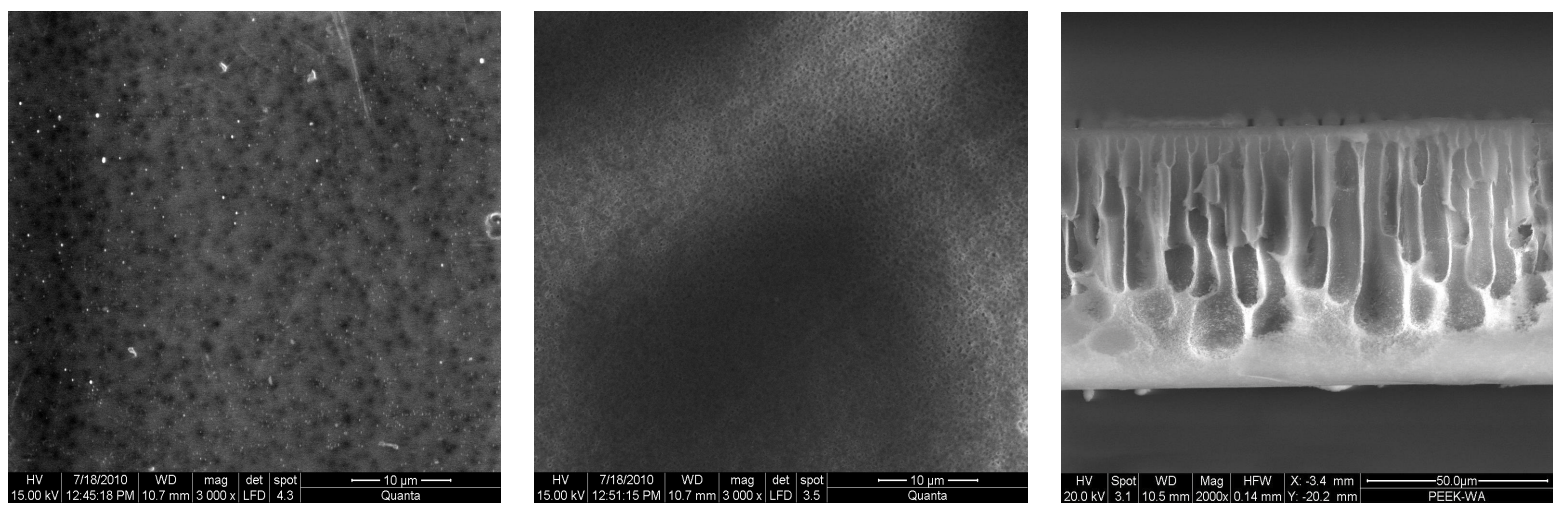

(a)
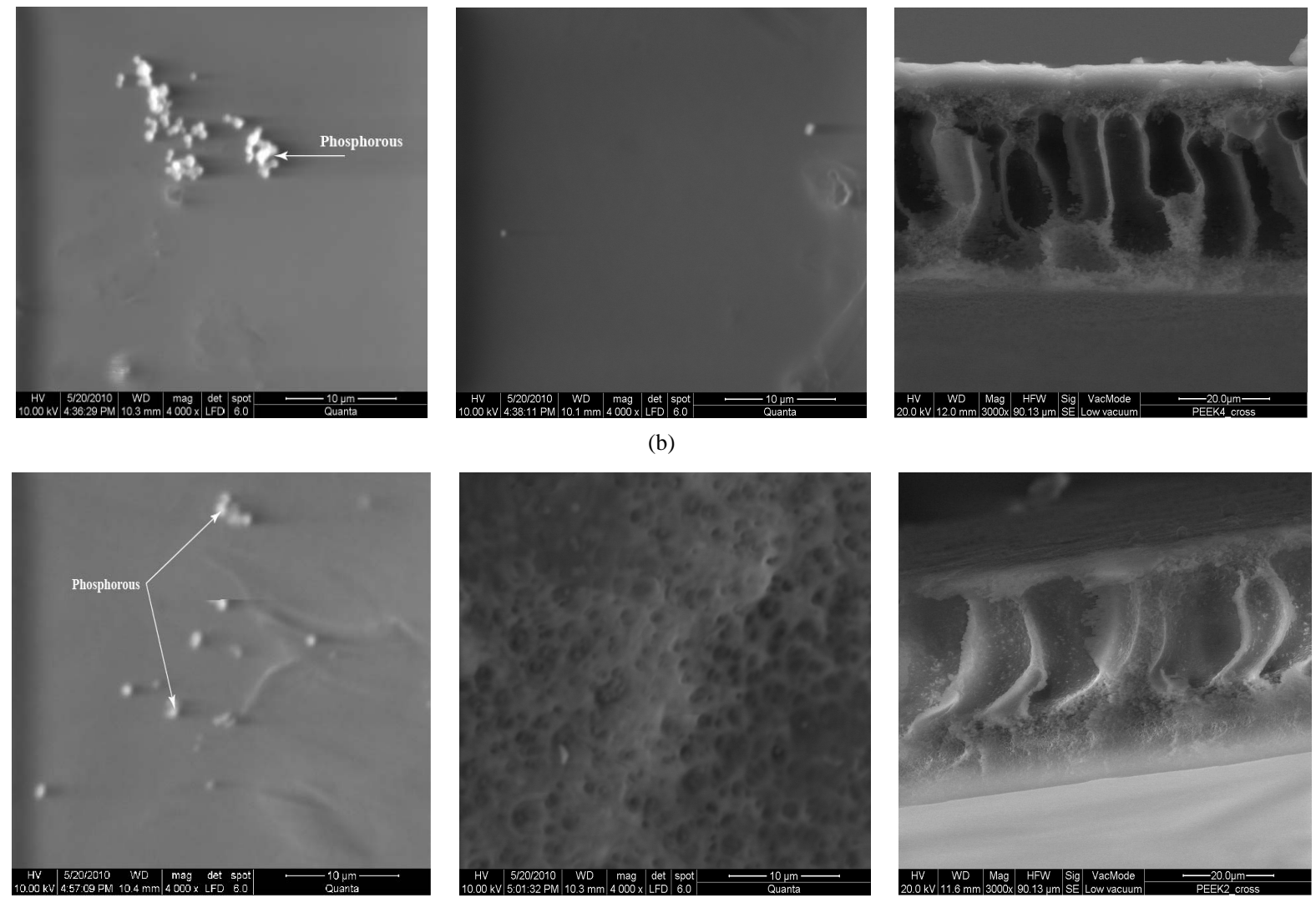

(c)
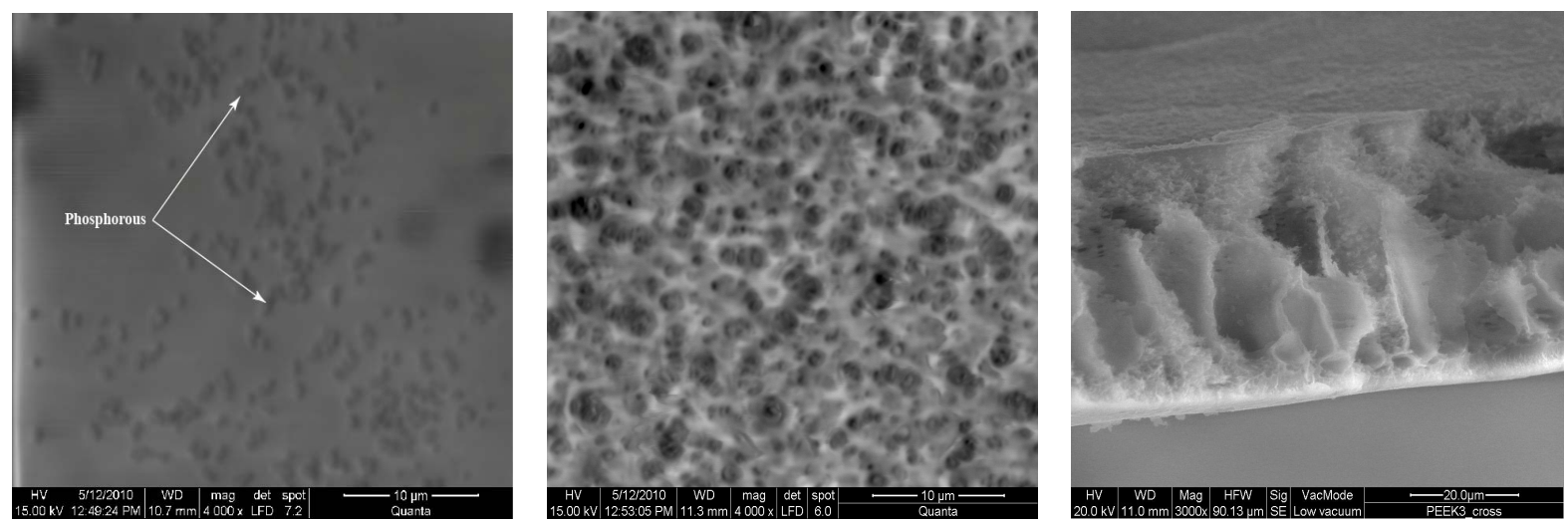

(d) 

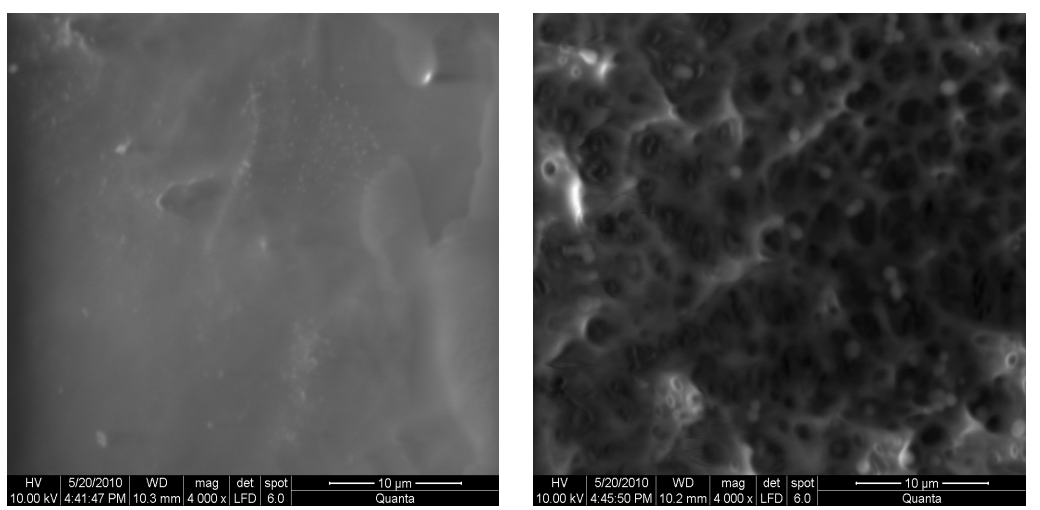

(e)
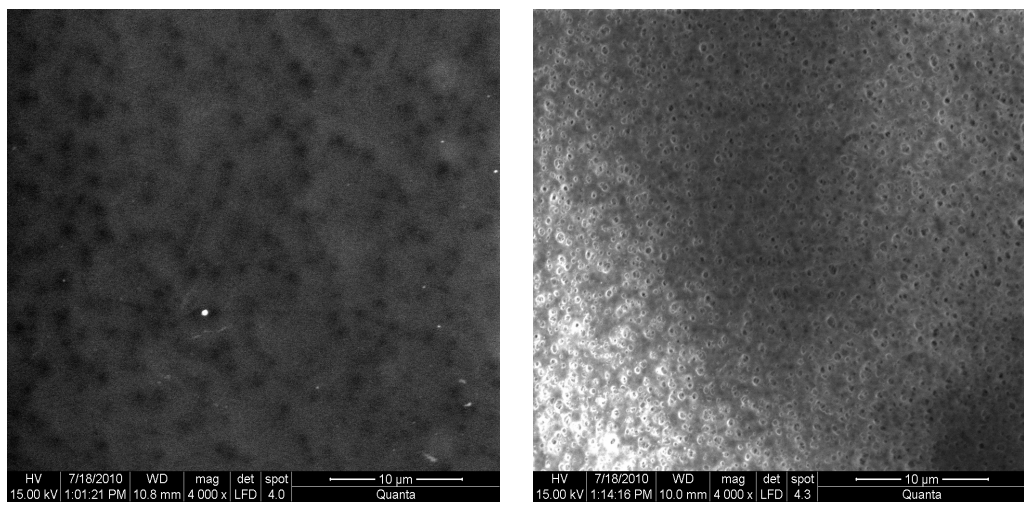

(f)
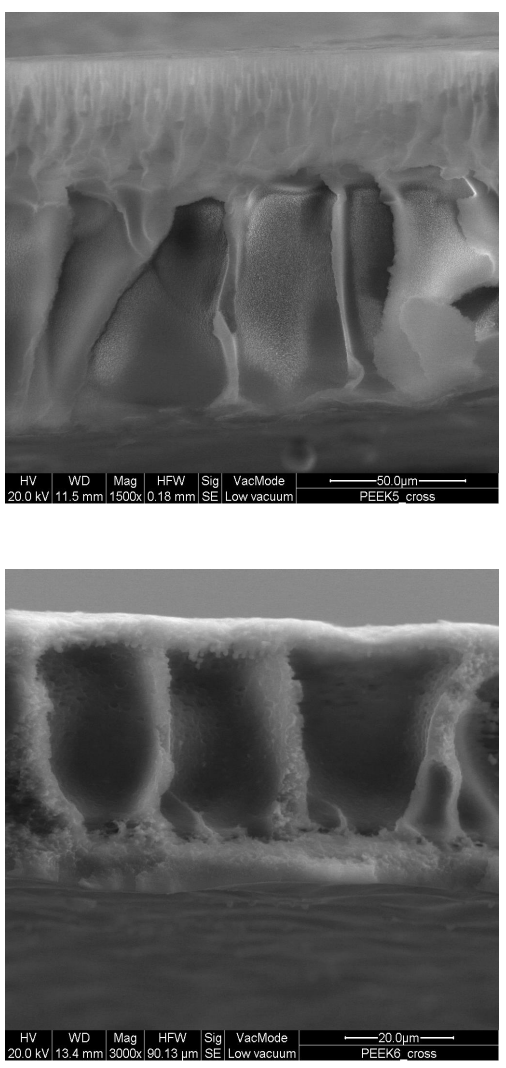

Figure 1. SEM images of the top, bottom surfaces and cross section of modified PEEK-WC membranes.

Table 2. Microanalysis of the top and bottom surfaces of the modified membranes.

\begin{tabular}{ccc}
\hline \multirow{2}{*}{ Membranes } & \multicolumn{2}{c}{ \% (Phosphorous) } \\
\cline { 2 - 3 } & Top surface & Bottom surface \\
\hline A & 00 & 00 \\
B & 11.25 & 2.5 \\
C & 00 & 00 \\
D & 5 & 00 \\
E & 3.82 & 3.82 \\
F & 00 & 00 \\
\hline
\end{tabular}

diffusing within the non solvent, by the high concentration of the polymer in the top surface and the low solubility of DEHPA in water. This technique can be used to modify membrane surface by desired molecules by wet phase inversion.

According to Fontas et al. [9], in the case of the very long evaporation step of the solvent in membrane preparation, the increasing of the carrier content enhances the interactions between the plasticizer and the carrier substituting the ones between the plasticizer and the polymer. As a result, a liquid micro-domain, where the carrier is solvated by the plasticizer, is created and the coalescence of the liquid micro-domain leads to the formation of continuous pathway connected between the two interfaces.

However, in asymmetric membranes the skin top layer plays an important role in the selectivity and transport properties of the membrane. As reported above, from micro-analysis results, this skin top layer can be modified by immersion precipitation by a suitable molecule (carrier) without plasticizers in order to avoid the dispersion of the carrier due to the interaction carrier-plasticizers. Figure 2 schematizes a proposed organization of the carrier in function of the presence of plasticizer.

\subsection{Thermal Properties}

To study the thermal properties of the ternary PEEKWC/DBP/DEHPA, we used TGA/DTA. Figure 3 presents the TGA data for the variance of the weight ratios in the PEEK-WC/DBP/DEHPA membranes at heating rate of $10^{\circ} \mathrm{C} / \mathrm{min}$.

The (TGA/DTA) data in Figure 3 show the thermal stability of the ternary membranes prepared by immersion precipitation method. The degradation temperature of PEEK-WC and remaining components are distinct.

Thermal degradation of virgin PEEK-WC, without additives, undergoes single stage degradation with a single 


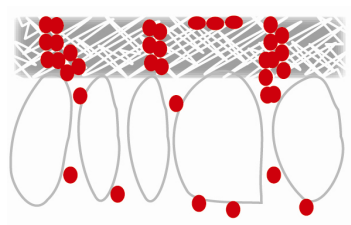

(a)

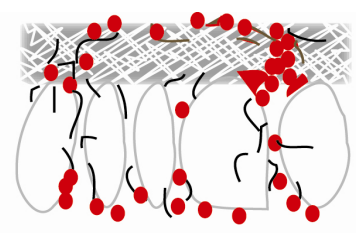

(b)
- Carrier

- Plasticizer

Figure 2. Schematic representation of the organization of the carrier in the membrane prepared by wet immersion-précipitation: (a) without plasticizer; (b) with the plasticizer.
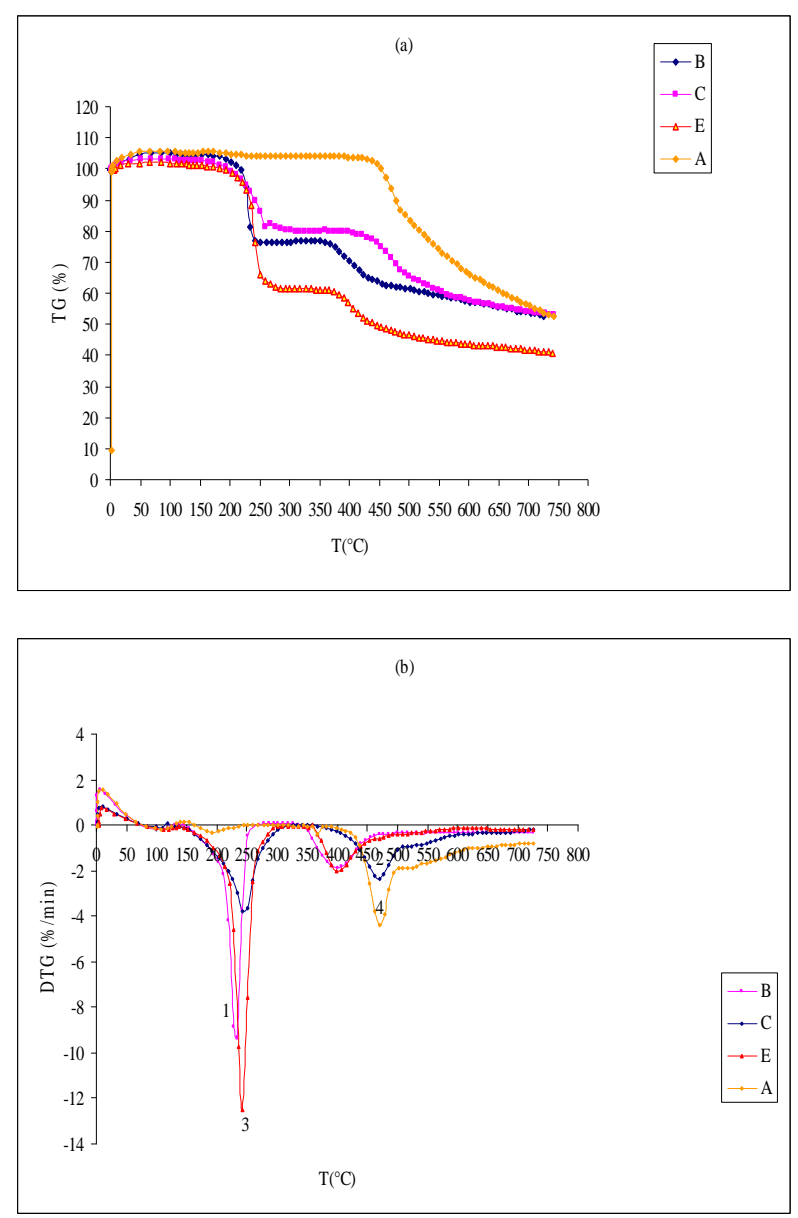

Figure 3. Thermal analysis of PEEK-WC membranes, containing DEHPA and DBP as additives, at different concentrations. (a) TGA; (b) DTA.

peak at around $480^{\circ} \mathrm{C}$, whereas the degradation temperatures of other samples are in the range of $200^{\circ} \mathrm{C}-250^{\circ} \mathrm{C}$. For PEEK-WC, the maximum weight loss of $40 \%$ occurs at $480^{\circ} \mathrm{C}$ due to thermal degradation of the PEEK-WC backbone. The TGA/DTA curves are considerably affected by adding dibutylphtalate (DBP) and di 2-ethylhexyl phosphoric acid (DEHPA). All the curves for the ternary system PEEK-WC/DBP/DEHPA undergo two- stage degradation at around $225^{\circ} \mathrm{C}$ and $425^{\circ} \mathrm{C}$.

The thermogram of the membrane (B) shows a twostage degradation for $25 \%$ around $225^{\circ} \mathrm{C}$ and $55 \%$ at $375^{\circ} \mathrm{C}$ loading from DEHPA degradation or evaporation (Boiling point closed to $200^{\circ} \mathrm{C}$ ) and polymer main chains respectively.

Adding the two components DBP and DEHPA, in the case of the membrane $C$ and $D$; the membranes have similar behaviour as the membranes containing DEHPA only. In others words, a two stage degradation is observed for that membranes.

The thermogram of the membrane $(\mathrm{C})$ undergoes two stages degradation at around $225^{\circ} \mathrm{C}$ and $475^{\circ} \mathrm{C}$ with weight losses of $20 \%$ and $55 \%$ due to the volatilisation of the mixture DEHPA and DBP, and the polymer backbone respectively. In addition increasing the concentration of DEHPA form $20 \%$ to $40 \%$ induce always a two stage degradation at $225^{\circ} \mathrm{C}$ and $330^{\circ} \mathrm{C}$ with weight losses of $40 \%$ and $60 \%$ respectively. The addition of DBP and DEHPA to PEEK-WC affect slightly the thermal stability of PEEK-WC membranes and suggest a lower interaction between the different components.

In the case of the ternary PEEK-WC/DBP/DEHPA system, the weight loss occurred after $200^{\circ} \mathrm{C}$ for all the membranes, which is high enough for using the prepared membrane in some applications such as lithium battery [10].

In fact, the role of the plasticizer on a polymer with many points of attachment along the polymer chain is to split the chains, break the attachment and shield the centres of forces that bind the chains together by selectively solvating the polymer at these points. However, the plasticizer molecules are not bound to the polymer chains; they are continuously attached and dislodged from these force centres. Because of the special backbone structure and branching of the plasticizer molecule, these compounds usually bring with them a large amount of free volume [11].

\section{Ni(II) Extraction by Supported Liquid Membrane}

Figure 4 shows the results of $\mathrm{Ni}(\mathrm{II})$ ions transport through supported liquid membrane using PEEK-WC as support and DEHPA (40\% volume) as carrier diluted in a mixture of heptane and xylene at different concentrations. We observed that the increase in xylene percentage in the mixture make the system more efficient in the transport of Ni(II) (Figure 4) but the stability is lower (after 1500 minutes, the loses are about 57\%). The presence of $x y-$ lene in the mixture probably makes the pore larger and the organic phase leave the pores easily by decreasing the capillary forces which retain the organic phase inside the pores. There is a steady increase in extraction efficiency 


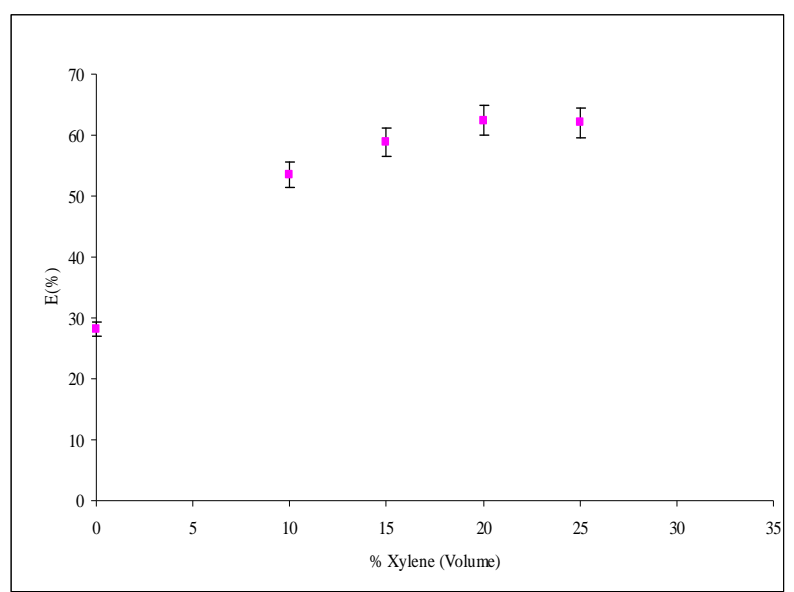

Figure 4. Influence of xylene concentration on extraction efficiency of $\mathrm{Ni}(\mathrm{II})$ through $\mathrm{SLM}$. Source phase: $\left[\mathrm{Ni}^{2+}\right]=60$ ppm, pH = 4.5 - 5; Stripping phase: $\left[\mathrm{H}_{2} \mathrm{SO}_{4}\right]=1 \mathrm{M}$; Membrane: PEEK-WC impregnated in $40 \%$ DEHPA diluted in a mixture of xylene and heptane; Flow stripping and source phases: $5.45 \mathrm{ml} / \mathrm{min}$.

with xylene concentration up to a level about $20 \%$ - 25\% $(\mathrm{V} / \mathrm{V})$ in heptane, after which more open structure is obtained and the feed solution pass through the membrane to the receiving phase.

\section{Solid-Liquid Extraction of $\mathrm{Ni}(\mathrm{II})$ by the Modified PEEK-WC Membrane}

Figure 5 shows the results obtained by solid-liquid extraction of $\mathrm{Ni}(\mathrm{II})$ by membranes based on PEK-WC, modified by DBP and DEHPA. We observed that the extraction efficiency increase by increasing DEHPA content, reaching $63 \%$ at $40 \%$ of DEHPA and $20 \%$ of DBP. However, the extraction efficiency decreases of about $50 \%$. This behaviour can be explained by the hydrophilic character of the PEEK-WC leads to high wettability of membranes by aqueous phase, combined to the highly asymmetric morphology of membranes. In fact, in the presence of DBP, a highly porous bottom interface is obtained insuring a highly contact area. Consequently, the extraction efficiency is enhanced (Figures 1, 4 and 5). Contrary to the membrane containing DEHPA alone at $40 \%$, the two interfaces, the top and the bottom surfaces, are smooth and dense (impermeable to water) reducing the contact area. Thus, the extraction efficiency is decreased.

\section{Conclusions}

In this study PEEK-WC flat sheet membranes with DBP and DEHPA as additives were prepared and characterized, using immersion precipitation technique. From SEM pictures, highly asymmetric flat sheet membranes were obtained with skin top layer and micro-porous

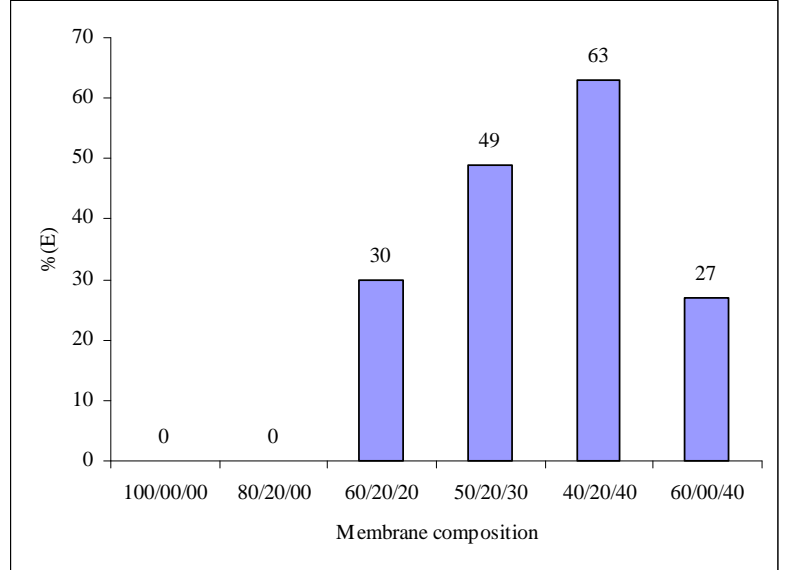

Figure 5. Solid-liquid extraction of Ni(II) by PEEK-WC flat sheet membrane modified by DBP and DEHPA. [Ni(II)] = $10 \mathrm{ppm}, \mathrm{T}=25^{\circ} \mathrm{C}$, Stirring: $600 \mathrm{rpm}$.

sub-layer. In addition, the presence of macro-voids and pores in the cross section was observed due to very low initial concentration of the polymer. The elementary analysis of phosphorus contained in DEHPA shows the tendency to aggregate in the top surface due to its low solubility and the exchange between the solvent and the non-solvent (water). However, the presence of the plasticizer (DBP) insures a dispersion of the DEHPA between the two interfaces and in the cross section.

The extraction of Ni(II) by supported liquid membranes using DEHPA as extractant, using asymmetric micro-porous PEEK-WC membranes, was very low due to the skin top layer. However, the presence of xylene in the organic phase (DEHPA and heptane) make the system more efficient which is in complete agreement with swelling results obtained varying the xylene percentage in n-heptane. Consequently, xylene allows a more open structure for PEEK-WC membranes reducing the capillary forces retaining the organic phase inside the pores.

In the case of PEEK-WC modified membranes by DBP and DEHPA, Ni(II) have been efficiently extracted from aqueous solutions, in particular in the presence of DEHPA and DBP due to the porosity of the bottom surface and the dispersion of DEHPA by DBP.

\section{REFERENCES}

[1] H. Myeong-Jin and B. Dibakar, "Changes in Morphology and Transport Characteristics of Polysulfone Membranes Prepared by Different Demixing Conditions," Journal of Membrane Science, Vol. 98, No. 3, 1995, pp. 191-200. doi:10.1016/0376-7388(94)00181-W

[2] R. E. Kesting, "Synthetic Polymeric Membranes. A Structural Perspective,” 2nd Edition, Wiley, New York, 1985.

[3] M. Mulder, "Basic Principles of Membrane Technology," Kluwer, Dordrecht, 1991. 


\section{doi:10.1007/978-94-017-0835-7}

[4] Y. Liu, G. H. Koops and H. Strathmann, "Characterization of Morphology Controlled Polyethersulfone Hollow Fiber Membranes by the Addition of Polyethylene Glycol to the Dope and Bore Liquid Solution," Journal of Membrane Science, Vol. 223, No. 1-2, 2003, pp. 187-199. doi:10.1016/S0376-7388(03)00322-3

[5] J. Xu and Z. L. Xu, "Poly(Vinyl Chloride) (PVC) Hollow Fiber Ultrafiltration Membranes Prepared from PVC/Additives/Solvent,” Journal of Membrane Science, Vol. 208, No. 1-2, 2002, pp. 203-212. doi:10.1016/S0376-7388(02)00261-2

[6] M. Sivakumar, D. R. Mohan and R. Rangarajan, "Studies on Cellulose Acetate-Polysulfone Ultrafiltration Membranes II. Effect of Additive Concentration,” Journal of Membrane Science, Vol. 268, No. 2, 2006, pp. 208-219. doi:10.1016/j.memsci.2005.06.017

[7] A. Idris, N. M. Zain and M. Y. Noordin, "Synthesis, Characterization and Performance of Asymmetric Polyethersulfone (PES) Ultrafiltration Membranes with Polyethylene Glycol of Different Molecular Weights as Additives," Desalination, Vol. 207, No. 1-3, 2007, pp. 324-339. doi:10.1016/j.desal.2006.08.008
[8] S. Bey, M. G. Bounomenna, M. Benamor and E. Drioli, "Preparation and Characterization of Microporous Membranes Prepared by Wet Phase Inversion. Application to Ni(II) Transport,” Materials Science Forum, Vol. 609, 2009, pp. 183-188. doi:10.4028/www.scientific.net/MSF.609.183

[9] C. Fontas, R. Tayeb, M. Dhahbi, E. Gaudichet, F. Thominette, P. Roy, K. Steenkeste, M.-P. F. Aupart, S. Tingry, E. T. Peyroz and P. Seta, "Polymer Inclusion Membranes: The Concept of Fixed Sites Membrane Revised,” Journal of Membrane Science, Vol. 290, No. 1-2, 2007, pp. 62-72. doi:10.1016/j.memsci.2006.12.019

[10] Y.-J. Wang and D. Kim, “The Effect of F127 Addition on the Properties of PEGDA/PVdF Cross-Linked Gel Polymer Electrolytes,” Journal of Membrane Science, Vol. 312, No. 1-2, 2008, pp. 76-83. doi:10.1016/j.memsci.2007.12.037

[11] M. Resina, J. Macanas, J. De Gyves and M. Munez, “Development and Characterization of Hybrid Membranes Based on an Organic Matrix Modified with Silanes for Metal Separation,” Journal of Membrane Science, Vol. 289, No. 1-2, 2007, pp. 150-158. doi:10.1016/j.memsci.2006.11.049 